\title{
Lexis
}

Journal in English Lexicology

$5 \mid 2010$

Lexicology \& Stylistics

Entre sémantique lexicale et sémantique textuelle : une analyse lexicale et stylistique de Before I Say Goodbye de Ruth Picardie

\section{Catherine Paulin}

\section{(2) OpenEdition}

\section{Journals}

Édition électronique

URL : http://journals.openedition.org/lexis/516

DOI : $10.4000 /$ lexis.516

ISSN : 1951-6215

Éditeur

Université Jean Moulin - Lyon 3

Référence électronique

Catherine Paulin, « Entre sémantique lexicale et sémantique textuelle : une analyse lexicale et stylistique de Before I Say Goodbye de Ruth Picardie », Lexis [En ligne], 5 | 2010, mis en ligne le 15 octobre 2010, consulté le 30 avril 2019. URL : http://journals.openedition.org/lexis/516 ; DOI : 10.4000/lexis. 516

\section{(c) (i) ()}

Lexis is licensed under a Creative Commons Attribution-NonCommercial-NoDerivatives 4.0 International License. 


\title{
Entre sémantique lexicale et sémantique textuelle : une analyse lexicale et stylistique de Before I Say Goodbye de Ruth Picardie
}

\author{
Catherine Paulin ${ }^{1}$
}

\begin{abstract}
This paper offers a double reading of a particular text Before I Say Goodbye (BISG) by Ruth Picardie: systemic and variationist, somewhere between language and discourse. The written text is close to orality: it is as set of exchanges of emails between Ruth Picardie and her friends, editorials published in the Observer Life columns and answers by readers. As the title clearly indicates, the isotopy is that of the end of life. The stance adopted here is that of the reception of the text: the target is to apprehend the impact that a particular discourse has on the co-construction of the meaning of lexical items in an analytical way.

The first part consists in a study of the semantic construction of lexical items which have undergone functional shift otherwise known as conversion. The point is to contrast the discourse value of lexicalised and perfectly stabilised denominal verbs to that of denominal verbs which are less commmonly used, less stabilised and which, therefore, have more to do with authorial expressivity.

In the second part, the idea is to distinguish between protopypical meaning in language and what could be prototypical meaning in a specific type of discourse. In BISG, the most recurrent construction of movement and perception verbs engenders recategorisation: the semantic bleaching of verbs such as feel, look, go and turn allows them to be in a relation with the existential truth of the subject.

The third part is devoted to sounds and rhythm and the construction of meaning in the discursive chain.

In as much as the systemic dimension of language is valued in the role it plays in the coconstruction of lexical meaning, the emphasis is put on the need to perceive and analyze the dialogical relation it has with the socio-historical dimension which is inbuilt in any speech act.
\end{abstract}

Keywords: co-construction of meaning - play on signifiers and sounds - lexical meaning discursive meaning - metaphor - functional shift - semantic shift

\section{Résumé}

Cet article propose une lecture entre système et variation, entre langue et discours, d'un texte particulier Before I Say Goodbye $e^{2}$ (BISG) de Ruth Picardie. Il s'agit d'un discours écrit proche de l'oralité : c'est un recueil d'échanges de courriers électroniques entre Ruth Picardie et ses amis, d'éditoriaux publiés dans Observer Life columns et de réponses de lecteurs. Comme le titre de l'ouvrage l'indique, l'isotopie est celle de la fin de vie. Le point de vue adopté dans l'article est celui de la réception du texte : l'objectif est d'appréhender, dans une démarche analytique, l'impact du discours sur la co-construction du sens d'unités lexicales. La première partie consiste en une étude de la construction sémantique d'unités lexicales par transfert catégoriel. Il s'agit de contraster la valeur en discours de verbes dénominaux lexicalisés à celle de verbes dénominaux qui relèvent davantage de la créativité de l'auteur.

\footnotetext{
${ }^{1}$ Université de Franche Comté - Besançon, Laseldi EA02281 : catherine.paulin@univ-fcomte.fr

${ }^{2}$ Penguin Books, 1998. Le titre est suivi d'un sous-titre « The bottom line is, I'm dying ».
} 
Dans la seconde partie, il s'agit de dégager le sens discursif le plus saillant d'unités lexicales verbales en discours. En effet, dans ce texte, la construction des verbes de mouvement et des verbes de perception engendre leur recatégorisation sémantique. La perte de poids sémantique lexical que connaissent les verbes feel, look, sound, go et turn dans BISG leur confère un rôle de cadrage du scénario verbal : ces unités verbales entrent en discours dans un rapport à la vérité du sujet qui devient son propre objet.

La troisième partie est consacrée au rapport entre phonie, jeux phonétiques et rythmiques et construction du sens dans la chaîne discursive.

Il n'est nullement question de rejeter la part systémique - du système langue - dans la coconstruction du sens lexical et textuel mais de la faire dialoguer avec la dimension discursive, sociale et historique inhérente à tout acte de parole, à toute production-réception de sens.

Mots-clés : co-construction du sens - corporalité - jeux de sons et co-construction - sens lexical - sens discursif - métaphore - transfert catégoriel - transfert sémantique 


\section{Introduction}

Le lexicologue s'intéresse aux mots de la langue, à la morpho-sémantique. Si l'on accepte l'idée selon laquelle le stylisticien aurait une approche du texte par ses formes, par toutes les strates qui font que les formes font sens, on accepte dès lors que la lexicologie et la stylistique s'inscrivent dans un continuum qui irait du plus systémique au plus discursif et textuel. Le propos de cet article se situe entre langue et discours. Les mots de la langue sont appréhendés dans le rapport qu'ils entretiennent avec le co-texte, la situation et le sujet de parole. La tension entre stabilité et flexibilité est au cœur de nos préoccupations comme l'est aussi le rapport nécessairement dialogique entre la pensée et la parole intérieures. Recevoir un discours, c'est intégrer la dimension sociale, historique de tout acte de parole ; c'est aussi recevoir la parole de l'Autre, qui peut être une fenêtre ouverte sur «ces autres moi » qui sommeillent en chacun de nous.

Nous nous sommes placée du point de vue de la réception du texte avec pour objectif de considérer l'impact qu'a le discours sur la construction du sens des unités verbales, sur les choix effectués et les effets pragmatiques produits. Une première remarque s'impose: l'expressivité, les phénomènes expressifs marqués sont sensiblement plus fréquents dans les courriers électroniques qui n'ont pas été l'objet d'un travail éditorial et sont très proches de la langue orale ${ }^{3}$. Nous partons de l'idée selon laquelle un état de langue neutre n'existe pas ou plutôt n'a qu'une existence théorique et du fait que l'énonciation individuelle ${ }^{4}$ constitue une manifestation de variations qui s'intègrent dans un discours particulier. Le discours et le genre dans lequel il s'inscrit imposent des contraintes, des choix, un style grammaticalisé que nous proposons de considérer comme intégré à la langue ou récupéré par celle-ci. Il s'agit donc de proposer une interprétation de faits de discours, en contexte, en prenant en compte ce qui relève du linguistique, du pragmatique, de l'énonciatif. Les notions d' « écart expressif », de " fréquence », de « registre » sont convoquées pour une " analyse variationniste » de la langue puisque l'objet auquel nous « essayons » notre analyse est la langue de l'individu dans un contexte particulier, dans ce qu'elle a d'idiosyncratique et de collectif. La langue de l'individu est elle-même dépendante de son environnement social et historique et n'est nullement stable. Nous rejoignons l'approche de Labov pour qui la variabilité est inhérente au système ${ }^{5}$. Selon Lecercle [1996: 189], il n'est pas de langue proprement dite, mais des rapports de force entre des variantes pragmatiques, dialectales :

Les mots peuvent être identiques, mais dans la bouche d'un enfant, d'un amoureux, ou d'un témoin dans un procès, l'énoncé n'est pas le même. Et il ne suffit pas d'écarter ceci en déclarant qu'il s'agit d'une variation « pragmatique ». Les mots ne sont que le véhicule d'un continuum de transformations sémantiques. Idiolectes et dialectes subvertissent la langue dont ils sont censés être des réalisations. La subversion réside dans le fait qu'il n'y a pas de langue proprement dite (autrement dit, pas de langue sans un reste qui la subvertit), mais un continuum de dialectes, en situation de « rapport de force » réciproque.

\footnotetext{
${ }^{3}$ On note la haute fréquence d'énoncés sans sujet : le sujet $I$ étant récupérable ou sous-entendu dans le contexte.

${ }^{4}$ Nous revenons rapidement sur ce concept car nous pensons que l'énonciation individuelle est nécessairement le produit d'interactions, que l'énonciation se construit dans son rapport à l'altérité, à l'état socio-historique dans lequel elle se produit. En bref, elle est nécessairement non seulement individuelle mais collective.

${ }^{5}$ Nous ne développerons pas ici une approche critique de la notion de système mais ajouterons simplement que la notion est à relativiser : il ne s'agit pas pour nous d'un état stable, mais toujours variable.
} 


\section{Construction du sens par transfert catégoriel et valeur en discours des verbes dénominaux : dégrés de lexicalisation}

Le corpus ${ }^{6}$ est le produit d'un relevé aussi exhaustif que possible des occurrences sur l'ensemble du texte ${ }^{7}$ : emails, éditoriaux et lettres de lecteurs. Travailler sur le verbe nous amène à contraster les catégories du nom et du verbe. Tous deux mots prédicatifs, le premier sert à dénommer un objet, une personne, un animal, une entité nationale, géographique, conceptuelle... tandis que le second sert à exprimer une action, un état, un devenir et se caractérise par une morphologie liée aux concepts de personne, de temps, de mode, parfois d'aspect. Selon le Dictionnaire Historique de la Langue Française ${ }^{8}$ :

[D]ans la langue grammaticale, verbum désigne le verbe, opposé au nom, vocabulum (=> vocable), opposition qui calque celle de rhèma / onoma en grec.

Se pose la question de la spécificité des verbes dénominaux par rapport aux autres verbes qui ne sont pas construits par conversion. Le verbe permet de mettre en relation différents rôles qui interviennent dans la conceptualisation de l'action ou de l'événement; le nom projette des propriétés ou qualia ${ }^{9}$ sur le verbe. Le verbe dénominal incorpore un argument - shadow argument dans la métalangue de Pustejovsky. Il a partie liée à la catégorie du verbe et à celle du nom. Plutôt que d'opposer les catégories du verbe et du nom, nous allons nous intéresser à ce qui les rapproche: le nom, comme le verbe, projette un de ses sens selon son environnement syntaxique et sémantique. Pustejovsky [1998 : 91] prend l'exemple des noms window et door et démontre que leur sens varie en fonction du rôle qu'ils jouent dans l'énoncé : figure (figure) ou fond (ground) :

(8) a. John crawled through the window.

b. Mary broke the window.

(9) a. Mary painted the door.

b. Mary walked through the door.

Les noms comme les verbes sont relationnels ; ainsi, dans les exemples sus-cités, les noms établissent-ils une relation entre les éléments constitutifs de l'entité ou entre la figure (figure) et le fond (ground). A ce titre, ils sont ambigus : ils peuvent renvoyer à l'objet, à une partie de celui-ci ou encore à l'une de ses caractéristiques. On reconnait bien là les associations logiques entre contenant / contenu : glass ; produit / producteur : press ; processus / résultat : examination; lieu / habitants : city, etc. L'ambiguïté est levée en fonction du contexte : le nom a potentiellement certaines qualia qui se réalisent ou non en fonction de sa structure argumentale. La structure en qualia appliquée au verbe permet d'appréhender la catégorie en termes de ses niveaux de représentation: nous faisons ici allusion à la classification sémantique selon le type et la structure de l'action ou de l'événement, la structure argumentale, les qualia ou qualités que le mot projette. Tant au niveau du verbe que du nom, il y a interaction entre structure argumentale et qualia.

\footnotetext{
${ }^{6}$ Voir annexe 1.

${ }^{7}$ A noter cependant que nous avons exclu de ce relevé les pages écrites par la sœur de Ruth Picardie, Justine Picardie ainsi que celles After Words écrites par son compagnon, le père de ses enfants Matt Seaton, très peu de temps après sa mort.

${ }^{8}$ Le Robert, Paris, 1994.

${ }^{9}$ Voir Pustejovsky [1998]. Nous renvoyons au chapitre 6 «Qualia Structure » dans lequel l'auteur définit, pages 85 et 86 , la structure en qualia selon quatre critères : 1 . Constitutif, à savoir la relation que l'entité a avec ses parties ou éléments constitutifs ; 2. Formel, à savoir ce qui distingue l'entité d'autres entités dans un domaine élargi ; 3. Télique, qui a trait au but ou encore à la fonction; 4. Agentif, qui a trait à l'origine.
} 
Les 54 occurrences de verbes dénominaux que j'ai relevées dans BISG présentent divers degrés d'expressivité en fonction de différents critères :

1. Degré d'usure : la conversion est plus ou moins " usée » au point parfois d'être tellement courante qu'elle n'est plus perçue, et perd donc de son expressivité au sens où tout écart par rapport à une norme intégrée n'existe pas.

2. Registre : le registre familier, le «parler cru» est vecteur d'expressivité supplémentaire. Dans les courriers électroniques, le lecteur est confronté à une forme d'oral scripturalisé.

3. Dimension métaphorique ou non du nom qui a fait l'objet de la conversion.

Pour une analyse de ces conversions de nom à verbe, je reprends la typologie de Tournier [1993 : 101-104] qui distingue quatre grands types de relation sémantique entre le nom et le verbe :

1. de création (prototype MAKE), soit près d'un tiers des conversions $\mathrm{N}>\mathrm{V}$;

2. de mouvement (prototype PUT ON, PROVIDE WITH, PUT IN, PUT INTO, REMOVE), soit près de $28 \%$ des conversions $\mathrm{N}>\mathrm{V}$;

3. d'instrument (prototype USE), soit environ un quart des conversions $\mathrm{N}>\mathrm{V}$;

4. de comportement (prototype BEHAVE), soit environ un huitième des conversions $\mathrm{N}>\mathrm{V}$ et enfin les autres types, soit environ un cas sur vingt, pour lesquels les relations peuvent être très diverses.

Ces données sont chiffrées à partir de données lexicographiques, hors contexte. L'échantillon sur BISG de 54 occurrences est certes très réduit mais proportionnellement important sur 91 pages ; ce qui est frappant est le renversement de la tendance constatée par Tournier en langue puisque les « autres types » comptent pour presque la moitié des occurrences (soit 26 sur 54); suit la relation de mouvement, soit près d'un quart des occurrences (soit 11 sur 54); puis la relation de comportement (soit 10 sur 54) et enfin la relation d'instrument (soit 7 sur 54). Ce qui est vrai dans le sytème de la langue ne l'est pas dans ce type de discours. Nous nous autorisons une forme de généralisation puisque nous avons travaillé sur quelques autres écrits intimes ou textes autobiographiques [Paulin : 2007, 2002, 2001] et cela, même si nous avons fait le choix dans cette contribution, de traiter d'un texte unique, dans son intégralité. S'en suivent quelques remarques : la forte proportion des autres types nous paraît en partie due au fait que c'est un sens métasémique du nom qui fait l'objet de la conversion. A titre d'exemples : book (8 à 13), face (23 à 28), peg (41), etc. Lorsque le nom converti en verbe est suivi d'une particule adverbiale (cas de conversion + composition), nous qualifions la construction de bicentrique, construction qui met en évidence l'incomplétude sémantique du verbe qui joue un rôle presque secondaire comparé à la particule adverbiale qui devient l'élément central qui prédique à propos du sujet : par exemple, block out (6), fob off (30), gun down (33), peter out (42), rule out (46), spirit off (48), etc. Lorsque la particule adverbiale joue un rôle d'intensifieur, elle permet à l'énonciateur un positionnement aspectuo-modal sur la relation sujet / prédicat : tel est le cas de end up (22) (+ renforcement de la télicité, qualia contenue dans le nom end). Le cas de la construction avec le pronom IT non référentiel en fonction objet illustre bien le fait que c'est la construction qui fait sens et non l'unité verbale seule, comme dans You can wear it after I've kopped it (16); I would love to have your underwear after you've copped it; The Movie would be great. Maybe you should make it after I've pegged it (41).

Pustejovsky [1998 : 56] écrit :

[...] the meaning is determined more by the context than by any inherent properties of the language lexicon. 
Il apparaît que ce ne sont pas seulement les propriétés sémantiques et syntaxiques des éléments lexicaux qui permettent leur polysémisation mais que celle-ci est facilitée par la connaissance extralinguistique qui joue, elle aussi, un rôle dans le changement de sens des unités, et, en fonction des différents contextes pragmatiques dans lesquels elles sont employées. Le contexte pragmatique facilite, voire déclenche, la polysémisation d'un mot ou d'une expression. De plus, nous ne pensons pas qu'il faille limiter l'importance du contexte aux seules relations intersubjectives mais plutôt qu'il faille l'élargir au type de texte, en fonction de ses isotopies, du genre dans lequel il s'inscrit. Le sens des constructions verbales nous semble donc être co-construit dans l'interaction des propriétés sémantiques des unités, du contexte pragmatique et du genre discursif et textuel qui s'inscrit nécessairement dans une société, à une époque donnée, dans sa dimension socio-culturelle et socio-historique. Nous plaidons pour la (ré)conciliation du sens lexical des unités, du sens constructionnel et du sens contextuel dans une acception large .

Pustejovsky [1998: 76] nous permet de tirer une première conclusion pour ce qui est des verbes dénominaux :

[...] all lexical items are relational to a certain degree, but the manner in which this property is expressed functionally will of course differ from category to category, as well as between semantic classes.

Le verbe dénominal, en incorporant un argument nominal à sa sphère, est particulièrement relationnel puisqu'il combine les relations et projections attribuables au nom incorporé et les relations actancielles attribuables à la catégorie du verbe.

Le texte BISG comporte d'autres types de conversion. Cependant, nous avons constaté que c'est toujours dans le domaine verbo-nominal que le transfert catégoriel est de loin le plus productif, avec 9 occurrences de conversion du verbe vers le nom sur 11 occurrences. Ceci confirme la tendance en langue.

La polarité verbo-nominale est complexe, y compris dans les cas de conversion $\mathrm{V}>\mathrm{N}$ et ceci même si les relations sémantiques possibles sont plus limitées que pour les conversions $\mathrm{N}>\mathrm{V}$. Dans le sens $\mathrm{V}>\mathrm{N}$, le nom désigne le plus souvent le procès : a lock-down (2), l'objet résultant: a little pay-back (5), l'agent du procès (pas d'occurrence dans BISG). L'interprétation des verbes dénominaux repose sur les propriétés sémantiques, physiques et culturelles de ce que désigne le nom ainsi que sur les propriétés actancielles du verbe. Dans les cas de conversion $\mathrm{V}>\mathrm{N}$, l'interprétation du nom repose essentiellement sur la classe sémantique du verbe de départ : activité, état, perception, etc., et aussi sur le rôle thématique que le nom a dans l'énoncé

Nous ferons une remarque ponctuelle sur un type de conversion mineur particule adverbiale $>$ V : Have you outed yourself? (4) Cette conversion de out (Adv vers V), loin d'être rare (1020 occurrences de " you have outed yourself » sur Google en 0,33 seconde) met en relief le rôle prédicatif saillant de la particule adverbiale dans go out. L'ajout d'un argument yourself co-référentiel du sujet grammatical permet un fort centrage sur celui-ci. L'ensemble de la construction confère un fort degré d'expressivité à l'énoncé.

Parmi les cas de conversion verticale qui ne sont pas lexicalisées, nous remarquons que ce ne sont pas des syntagmes figés mais des relations prédicatives, séquences fortuites, nominalisées ou en position pré-nominale (what-surname-to-give-the-kids dilemma, p. 59), qui sont les plus fréquentes. 


\section{Sens constructionnel des verbes de perception : feel, look, sound et des verbes de mouvement : go, turn $^{10}$}

La récurrence de périphrases verbales est frappante : leur complexité permet de construire le sens avec expressivité et est adaptée à la caractérisation du sujet, terme que nous prenons ici au sens de sujet grammatical et de sujet humain. La très grande majorité des énoncés comporte un sujet grammatical animé humain, instancié ou non, le plus souvent le pronom personnel de la première personne du singulier. Ces périphrases verbales ne sont cependant pas inédites mais bel et bien grammaticales : l'expressivité apparaît comme inscrite dans la grammaire de la langue qui permet de faire des choix en fonction des opérations de la pensée que le locuteur souhaite révéler, plus ou moins consciemment. Les périphrases verbales permettent au locuteur de re-construire le sens : contrairement à ce qui se passe avec les verbes simples, l'arbitraire n'est pas absolu, la construction est en quelque sorte motivée. Les périphrases signifient en analysant ce qui n'équivaut pas à dire que leur sens se déduit de la somme des sens des éléments constitutifs, loin s'en faut.

Every grammatical construction encodes a certain meaning, which can be revealed and rigorously stated, so that the meanings of different constructions can be compared in a precise and illuminating fashion, both within one language and across language boundaries.

Grammar is not semantically arbitrary. On the contrary, grammatical distinctions are motivated (in the synchronic sense) by semantic distinctions; every grammatical construction is a vehicle of a certain semantic structure; and this is its raison d'être, and the criterion determining its range of use. [Wierzbicka 1988: 3]

I maintain that grammatical constructions embody certain meanings; and I try to reveal these meanings, to show exactly what they are, and how the use of a given construction can be predicted from its meaning. [Wierzbicka 1988: 7]

A propos des verbes de perception et de mouvement qui ne «disent » ni la perception sensorielle ni le mouvcment - c'est l'écrasante majorité des cas dans ce texte, à tel point que nous ne pensons pas avoir remarqué d'emploi «littéral » de feel - nous voudrions aborder la notion de subjectification telle que la définit Langacker [1990 : 315-341]. Nous préférons conserver le terme de subjectification dans un premier temps afin d'éviter l'ambiguïté que la traduction pourrait introduire. Cependant, et par commodité, nous parlerons ensuite de subjectivation. Nous partons de l'idée selon laquelle le sens d'une expression - verbale pour ce qui nous concerne - ne peut être réduit à une caractérisation objective d'une situation. La façon dont l'énonciateur construit la situation et la décrit à des fins expressives est particulièrement importante pour le sémanticien [Langacker 1990: 315]. Nous nous attacherons dans les remarques qui suivent au regard que l'énonciateur porte sur la scène, à la manière dont il se situe par rapport au fond - dans le sens de ground chez les cognitivistes. C'est par rapport au point de vue, c'est-à-dire à la position qu'a l'énonciateur par rapport à la scène, que le co-énonciateur «calcule » le degré de subjectivité ou d'objectivité de ce qui est prédiqué. Le terme subjectif est employé lorsque le sujet expérientiel, l'individu qui perçoit, fait partie de la situation, du fond (ground) sans être en mesure de pouvoir «objectiver » au sens de se détacher de la figure (figure), d'avoir une vision extérieure. Langacker développe l'idée selon laquelle plus «l'individu percevant» est immergé dans l'expérience de perception qu'il a, plus il perd conscience de lui-même et de l'angle duquel il perçoit. De ce

\footnotetext{
${ }^{10}$ Le corpus est donné en annexe 2.
} 
fait, l'objet de la perception est «subjectivé » car très fortement lié au "sujet percevant ». Nous reviendrons sur ces notions dans le contexte textuel et thématique particulier qui nous occupe ici. Avant cela, il nous paraît nécessaire d'ajouter que les notions d'objectivité et de subjectivité ainsi définies sont applicables à toute situation : aucune situation d'énonciation ne saurait être dénuée de point de vue. Nous partons de l'idée selon laquelle le choix de l'unité verbale et de la construction permet de faire fluctuer le degré de subjectivation. La difficulté réside dans le fait que l'on ne voit vraiment et que l'on ne donne à voir que ce par rapport à quoi on se positionne verbalement en tant que sujet. Il semblerait que nous établissions entre les verbes de perception et de mouvement et leurs emplois "non littéraux » un rapport de vérité : celle du sujet qui, en choisissant ces verbes non "neutres » et en se situant sur la scène, devient l'objet de son dire. Nous impliquons ainsi qu'il n'y a pas de réalité du sujet pour lui-même sans subjectivation préalable. Nos objets ont-ils une existence, une réalité s'ils ne sont pas reliés à nous-mêmes? La perte de poids lexical (semantic bleaching) que connaissent les verbes feel, look, sound, et aussi go et turn dans BISG leur confère un «poids sur le fond $»$ : ils prédiquent à propos du sujet qu'ils placent sur la scène et qui devient son propre objet. Nous sommes d'accord pour penser que le verbe non tensé, à un mode non fini, dit un type de procès; que le verbe à un mode fini, affecté des marques de personne et des marques aspectuo-temporelles, situe le procès dans le temps et par rapport à une situation. Le temps, l'aspect, la modalité ont trait au fond. Il nous paraît raisonnable de penser que les métasémies verbales aussi : qu'elles permettent non seulement de dire un type de procès mais qu'elles permettent aussi de le situer par rapport à l'énonciateur. La grammaticalisation - au sens large - des verbes à laquelle nous faisons allusion en évoquant la notion de semantic bleaching leur confère non plus tant une valeur processuelle qu'une valeur de grounding predicators. Nous adoptons ici l'expression de Langacker de true grounding predicators [Langacker $1990: 321]$ :

We can reasonably suppose that only "grammaticized" (as opposed to "lexical") elements can serve as true grounding predications. For the moment, it is sufficient to note that a grammaticized element is quite schematic semantically (i.e it lacks the specificity and rich detail typical of lexical items) and tends to assume a "relavistic" or "topological" character rather than indicating a specific shape or value (cf. Talmy 1988b).

Ce haut degré de subjectivation va de pair avec l'évolution sémantique des verbes de perception et de mouvement. Ainsi, on passe du plus lexical au plus grammatical sans qu'il y ait de rupture. La perte de poids sémantique de ces verbes est certes relative, et, de plus, elle s'accompagne du fait que le sens propositionnel se charge «d'expressivité textuelle »: il ne s'agit plus de prédiquer ou de décrire une situation extérieure au sujet percevant mais de l'évaluer de l'intérieur, voire de l'intérioriser. Ce double processus de subjectivation objectivation permet au sujet d'évaluer et d'intérioriser ce qui lui arrive en en faisant son « objet subjectivé ». Nous touchons bien ici au textuel, à l'écriture de l'intime, au contextuel thématique. Il nous semble que nous passons aussi de la subjectivation au sens étroit à la subjectivation au sens large, à l'émergence du sujet qui fait de lui-même son objet verbal.

La décoloration sémantique des verbes feel, look, sound, go et turn permet d'expliquer, dans une large mesure, la récurrence de leur emploi en tant que copules dans BISG. Nous avons relevé 27 occurrences de la construction $\mathrm{S}-\mathrm{V}$ - Adj. prédicatif à la $1^{\text {ère }}$ personne contre 5 occurrences seulement à la $2^{\text {ème }}$ personne et 5 occurrences à la $3^{\text {ème }}$ personne. La prépondérance de la $1^{\text {ère }}$ personne tient au fait qu'il s'agit « d'une mise en scène du moi ». Le verbe feel est très largement dominant. En revanche, parmi les 5 occurrences de la $2^{\text {ème }}$ et de la $3^{\text {ème }}$ personne, on remarque que look est employé deux fois à la $2^{\text {ème }}$ personne, une fois à la 
$3^{\text {ème }}$ personne (sur 7 occurrences) et que sound n'est employé qu'à la $2^{\text {ème }}$ personne ( 1 occurrence) et à la $3^{\text {ème }}$ personne (1 occurrence). Nous en déduisons que le verbe feel facilite l'objectivation du sujet par lui-même plus que les deux autres verbes de perception look et sound. Dans les énoncés à la $2^{\text {ème }}$ et à la $3^{\text {ème }}$ personne, l'énonciateur sur la scène est le point d'ancrage subjectif à partir duquel la caractérisation du sujet grammatical est opérée, à titre d'exemples :

I guess most of my friends simply feel desperately sorry for Matt, me and the children (p. 73) ; You look so well (p. 48), But your house always looks fabulously chic and clean (p. 25); You didn't sound your normal chipper self (p. 19), All this (complementary alternative treatment) may sound ridiculous (p. 76).

L'emploi du verbe copule BE ne permettrait pas de placer l'énonciateur sur le même plan que le sujet grammatical dans le fond. Avec BE, la caractérisation du sujet grammatical ne serait pas cependant totalement neutre, ce qui tient à la valuation que posent l'adjectif prédicatif ou encore d'autres modalisateurs lexicaux ou grammaticaux ou quantifieurs tels que so, desperately, simply, fabulously, a bit, less.

Avec un verbe de mouvement employé pour indiquer le changement d'état, l'énonciateur conceptualise subjectivement le changement d'état du sujet grammatical. Selon l'unité verbale et la construction - avec particule adverbiale ou syntagme nominal prépositionnel - il y a ou non télicité. La subjectivation du changement d'état tient au fait que l'énonciateur localise mentalement le chemin parcouru entre un état initial et un état ultérieur (atteint ou non) par rapport à un point de référence qui fait partie du fond :

The foregoing instance of subjectification represents a general type characterized by the following central property: spatial motion on the part of an objectively construed participant is replaced by subjective motion (mental scanning) on the part of the conceptualizer. [Langacker $1990: 327$ ]

The directionality inherent to these latter expressions, and the sense of "movement" they inspire, can only be attributed to subjective motion by the conceptualizer, who traces a mental path by scanning in a particular direction along the subject's expanse. [Langacker $1990: 328$ ]

Les énoncés construits avec un verbe de mouvement-changement d'état concernent presque tous l'énonciateur co-référentiel au sujet grammatical $I$. Lorsque le sujet grammatical n'est pas co-référentiel au sujet-énonciateur, le double processus subjectivation - objectivation dont il a été question nous paraît se répéter. Prenons un exemple : [...] the treatment turned into a huge pressure (p. 77). L'unité verbale, la construction verbale ainsi que la présence d'éléments lexicaux modalisants convergent pour indiquer que le sujet grammatical est perçu comme "mouvant» par le sujet-énonciateur qui suit sa trajectoire. Dans l'énoncé de la page 38, the diagnosis turns you into a grumpy, bitter, envious old cow, le pronom générique you masque et signale en même temps que le changement est calculé par le sujet-énonciateur qui est inclus dans la sphère du sujet grammatical. La construction confère une valeur causative à l'unité verbale.

Dans BISG, l'emploi qui est fait des verbes de perception et des verbes de mouvementchangement d'état est certes conforme à ce que le sytème-langue permet mais ce sont les emplois dits "dérivés », par extension d'un sens qui serait premier, qui sont, d'une part, les plus récurrents et qui, d'autre part, caractérisent ce type d'écriture. Le double mouvement subjectivation - objectivation permet à l'individu de devenir son propre objet : en ce sens, 
l'écriture permet peut-être une forme de détachement du sujet devant son objet, ici la mort. Au niveau des constructions, le complément prépositionnel, dans lequel la préposition joue le rôle prédicatif prépondérant, permet de préciser l'état ou l'aboutissement du changement d'état du sujet grammatical perçu subjectivement par le sujet-énonciateur. La préposition INTO qui, selon notre relevé est systématiquement employée dans BISG avec le verbe turn, ajoute en termes de télicité au sémantisme du verbe. Dans les constructions V + Adj., V + syntagme nominal, la complémentation joue toujours le rôle prédicatif essentiel. L'unité verbale continue cependant d'assurer la référence au procès qui est précisé notionnellement par la complémentation. Tesnière [1996 : 159] nous renseignait déjà à ce propos : c'est le complément qui «fait fonction de verbe » et c'est le verbe qui «sert à marquer le rôle verbal » du complément.

Pour ce qui est des constructions propositionnelles, avec le verbe composé $(\mathrm{V}+$ part. adv.) turn out, dans les deux occurrences de construction propositionnelle : infinitive en TO et proposition à mode fini (aux pages 27 et 29 du texte), l'ensemble de la construction confère à l'unité une valeur de « verbe de survenance » :

Interviewed Robbie (to his friends) for Style; became slightly in love. He turned out to have shrunk. (p. 27)

Well, after weeks of hassling (by me) and faffing (by Guy's) it turns out I do have skull 'involvement'. (p. 29)

La prédication principale est à l'extérieur du verbe qui, quant à lui, permet à l'énonciateur d'ajouter un commentaire modal - épistémique - sur son contenu. Le verbe et l'ensemble de la construction condensent des significations modales et pragmatiques qui s'établissent entre le sujet énonciateur, le sujet grammatical et la complémentation propositionnelle. Dans le cas où IT est support de prédication (p. 29), c'est la maladie, auquel renvoie l'euphémisme skull 'involvement' qui fait l'objet du positionnement de l'énonciateur.

La complémentation propositionnelle du verbe feel nous amène à faire une remarque relative au fait que, d'une part, perception et connaissance sont intrinsèquement liées, et, d'autre part, le sens de l'unité est largement conditionné par le contexte discursif et textuel : le verbe en soi n'a que peu de "matière notionnelle », son sens tient aux relations qu'il permet entre tous les éléments de la situation discursive, pragmatique et textuelle. Nous touchons ici à un paradoxe : d'un côté, le sens dit lexical est davantage variable que le sens grammatical qui est lié à la catégorie et qui a quelque chose de plus stable et de plus universel, mais, en même temps, la plasticité du sens lexical tient aux constructions grammaticales qui existent en puissance dans les lexèmes :

The meaning of an expression is the result of integrating the meaning of the lexical item into the meanings of constructions. [Pustejovsky $1998: 58$ ].

Nous soulignons l'emploi du pluriel meanings of constructions : les constructions en général et les constructions propositionnelles en particulier sont polysémiques même si l'on peut accepter qu'à une construction propositionnelle "correspond» un sens prototypique. De plus, le contexte textuel et la connaissance que le lecteur a de la situation jouent le rôle de déclencheur de l'acception qu'il a des unités verbales et des constructions qui sont toutes deux pragmatiquement motivées. Le verbe et la construction contribuent à générer une polysémie qui est relationnelle et logiquement organisée. Avec feel, on oscille entre «je sens » et « je sais quelque chose » et «quelque chose me dit que ». La notion verbale feel nécessite au minimum un expérient qu'il soit exprimé ou non, et une entité perçue, qui est contenue dans la complémentation propositionnelle et fait l'objet du compte-rendu de l'expérience. 
L'intentionnalité liée à l'unité feel implique que la perception du sujet expérient n'est pas l'aboutissement d'un choix. Feel permet de mettre en tension le sujet expérient et l'objet de la perception ou de la connaissance qui s'impose à lui. Ces propos sont à revoir lorsque feel est construit avec FOR, dans feel for something, le sens de la préposition confère une valeur de visée à l'ensemble de la construction. Feel est de très loin le verbe de perception-connaissance intentionnel sans visée le plus employé, ce qui nous paraît coller avec la thématique du texte.

Nous poursuivons l'idée selon laquelle la thématique et l'écriture intime imposent l'emploi de périphrases verbales, de certaines constructions plutôt que de verbes simples. La complexité de la périphrase permet de mieux construire, avec une force expressive accrue, ce que le sens a d'individuel et aussi d'inscrit dans le collectif sociétal. Les verbes de perception lorsqu'ils sont construits avec AS IF ou avec LIKE + GN, permettent d'énoncer la perception de ce qui n'est pas, de ce qui est virtuel mais construit par rapport au sujet-énonciateur qui, ainsi, dit toute la dimension subjective de sa propre réalité. C'est ce que le récepteur entend page 54 :

Much better than Mogadon, which makes you feel as if you've been hit over the head by a frying pan.

L'interprétation de feel like est comparable : la construction ne fait que poser la tension entre un sujet et un objet sans dire quoi que ce soit de l'actualisation de celui-ci. Le verbe et la construction permettent un travail modal sur la relation prédicative : ils ne catégorisent pas la référence mais permettent un positionnement subjectif, appréciatif de l'énonciateur. Look et sound LIKE n'informent pas quant à la "réalité objective » du sujet grammatical mais à la manière dont il est perçu. La construction «subjectivise »- le point de référence étant l'énonciateur - des relations pragmatiques co-construites par les pronoms, le type d'énoncé les énoncés interrogatifs ou injonctifs en particulier - le lexique appréciatif.

Nous cherchons à souligner la prégnance du discours, de sa thématique, du récit intime sur la construction du sens des verbes et des constructions verbales par le récepteur. Nous avons affaire à une sorte de "micro-système ». L'analyse repose sur la réception en un temps $\mathrm{T}$ du discours mais cette approche synchronique ne doit cependant pas nier le fait que toute production langagière est à situer dans un contexte socio-historique. L'interprétation de certaines locutions verbales telles que go nuts, go bonkers, be dotty, kick the bucket, etc. repose sur le fait que nous avons intégré leur histoire, celle de leur charge métaphorique, et qu'elles font sens en bloc, en tant que telles. D'autres locutions verbales comme let go (of sth), be up to sth, keep so going, be on the mend semblent typiques de l'époque dans laquelle nous vivons et du centrage sur l'état psychologique des individus qui la caractérise. L'ensemble de ces locutions illustrent parfaitement la non-compositionnalité du sens qui émerge de la construction comme du contexte discursif, textuel et socio-historique.

\section{Métaphores, hypallages, rythme, son et construction du sens}

Nous ne traiterons pas des verbes dénominaux métaphoriques que nous avons mentionnés dans le point 1. L'ensemble des métaphores concourt à donner une couleur stylistique particulière au texte. Comme nous l'avons signalé, leur force expressive est inversement proportionnelle à leur degré de lexicalisation et d'usure. Nous nous en tiendrons ici à quelques remarques relatives à des éléments métaphoriques autres que les verbes dénominaux et aux hypallages du texte pour lesquels la question du degré de lexicalisation et d'usure se pose. Nous préciserons les liens que nous établissons entre style et force expressive. Retenons deux séquences hypallagiques : 
Don't you love the way journalists lie: 'dusty' pink? How about screaming fuchsia? And, 'she intends to redecorate with a more subtle bone-coloured scheme'... (p. 19)

Dusty pink est une collocation si bien intégrée à la langue que l'usager n'a plus guère conscience de sa dimension hypallagique. L'expression screaming fuchsia qui repose sur une métaphore intersensorielle est, elle aussi, intégrée, mais dans une moindre mesure et elle jouit de facto d'une plus grande force expressive. De la même manière, et à titre d'exemples, les métaphores verbales suivantes confèrent une dimension expressive au discours même si elles ne constituent pas un écart par rapport à la norme :

I wish summer would hurry up: I never know what shoes to wear in the winter. (p. 26)

[...] when he peacefully slipped away. (p. 63)

Dans l'exemple suivant (p.72), d'un niveau de langue familier, dans lequel le pronom IT n'est pas anaphorique au sens strict (pas de reprise endophorique), nous notons qu'en dépit d'une forme de figement de la collocation ${ }^{11}$ qui n'est pas un hapax, la force expressive de la relation $\mathrm{S} / \mathrm{P}$ my former niceness / cut it reste intacte :

I can't believe my popularity is simply due to the fact that I'm not yet looking as scary as my fellow patients... Nor can it be my former niceness, which never used to cut it when turning up for birthday dinners. (p. 72)

Métaphores, hypallages, périphrases verbales, même si elles sont stabilisées - comme go nuts, go bonkers, go a bit bonkers, be dotty, be a bit dotty, be up to (something), catch sight (of something), keep someone going, be on the mend, qui sont toutes présentes dans BISG contribuent à dynamiser le rapport entre expression et expressivité. Nous avons employé ce concept imprécis à plusieurs reprises. Son imprécision nous semble liée au fait qu'il est employé tant pour ce qui relève de la dimension stable du lexique que de la dimension plus variable qui relève des choix discursifs et stylistiques d'un locuteur-énonciateur. Cependant, ce sont plus particulièrement les métaphores et hypallages "vives" du texte, non encore usées par une (très) haute fréquence d'emploi, qui sont signifiantes non seulement pour ce qu'elles disent mais aussi, et peut-être plus encore, parce qu'elles « ont l'air de vouloir dire quelque $\operatorname{chose}^{12} »$. La distinction entre dire et vouloir dire nous paraît essentielle. Nous souhaitons ajouter que même les mots du lexique stabilisé, institué ont trait, dans leur dimension métasémique ou connotative, au vouloir dire du locuteur-énonciateur. Nous sommes là du côté de la réception, du sens virtuel qui est à recontruire, qui demande de nous entrevoyions, pénétrions le vouloir dire du locuteur-énonciateur. Dans BISG, l'expression vise l'expressivité : elle n'est pas utilitaire, destinée à dire un contenu nouveau. Le style, les choix lexicaux constituent l'essentiel du discours : son vouloir dire. Quand il s'agit de vie humaine ou de fin de vie, c'est l'indicible, ce vouloir dire caché, qui recèle le sens. Le recours - conscient ou non - à des lexies construites - par conversion ou par métasémie - des

\footnotetext{
${ }^{11}$ Le nom niceness attire not cut it. Nous avons relevé sur Internet dans un registre comparable à BISG : «Why is it that niceness alone doesn't seem to cut it for anyone? [...] For every nice guy who has been turned down by a woman there's undoubtedly a nice girl who's been turned down by a guy... », in "The Problem with Niceness », Newageknight, Thu May 7, 2009. Le surlignement est le nôtre comme dans les passages de BISG que nous citons.

${ }^{12}$ Nous empruntons cette expression à Ruyter Raymond [1955], "L'expressivité », Revue de métaphysique et de morale : 69-100. Nous devons cette référence à Philippe Monneret dans son introduction à La fonction expressive, volume 2, à paraître dans Recherches en linguistique aux Presses Universitaires de Franche-Comté.
} 
constructions polylexémiques, plutôt qu'à des lexies simples à partir desquelles aucun processus lexicogénique ne pourrait être inféré, permet que se surajoute le vouloir dire du locuteur-énonciateur à la réalité des états, des actions, des événements.

Les sons et le rythme possèdent aussi un pouvoir d'expression et d'expressivité. Le sens s'attache non seulement aux unités morphématiques mais également aux phonesthèmes. Les morphèmes et les phonesthèmes ont des caractéristiques communes en ce qu'ils ont trait aux connotations, au pouvoir évocateur - autre que dénotatif - des mots qui permettent au sujet d'inscrire son vouloir dire dans son discours. Le rythme, en ce qu'il incarne «l'organisation du mouvement de la parole » [Meschonnic 2007 : 45] est lui aussi expressif : le corps de la langue dépasse les unités-mots. C'est dans la corporalité langagière que s'inscrit une grande part de l'expressivité du sujet dans un style qui le caractérise. En ce sens, les répétitions lexicales - ou syntaxiques - les séries prosodiques, les ruptures... sont signifiantes. Prenons quelques exemples :

1) Répétition lexicale, écho, mise en abyme, effet de vertige :

A bearded, yoga-practising Kathy Acker type would say that it's marching on because the chemo compromises your immune system, but it's pretty compromised anyway, otherwise I wouldn't have cancer. (p. 12)

2) Récurrence de phonèmes et pouvoir évocateur des sonorités :

Well, after weeks of hassling (by me) and faffing (by Guy's) it turns out I do have skull 'involvement'... (p. 29)

Supposed to go on a 'mental journey' while holding hands and listening to new age bonging, but all I could think about was abdominal bloating. (p. 30)

So now I have strict instructions that if anyone asks I am to say you are soon to be blooming with rude health. She is bonkers as conkers of course, but possibly right. (p. 30, il s'agit ici de paronomase).

3) Polysémie, ambiguïté et sons :

Isn't it so utterly bamboozling to discover that something so beautiful, fantastically talented and unique as me or you depends on some clump of dysfunctional plasma - balls or calcium concentrates. (p. 29, bamboozling, qui n'est pas un hapax, a ici le sens de « confusing »)

Selon Meschonnic [1982: 100],

Les rythmes sont la part la plus archaïque dans le langage. Ils sont dans le discours un mode linguistique pré-individuel, inconscient tout comme le fonctionnement du langage.

Et pourtant, le rythme, qui est presque étranger au sujet, en s'imposant à lui, lui permet de le faire sien et de se révéler. A ce carrefour entre le discours intentionnel et le discours " involontaire », apparaissent métaphores, visions, néologismes qui, loin de " ne pas parler », sont non seulement intelligibles mais encore chargés d'expressivité. Le texte de BISG, son style proche de l'oralité dans des échanges électroniques ou épistolaires rédigés dans une langue familière, urbaine et bien d'aujourd'hui nous offre des jeux sur les sons chargés de signifiance. 


\section{Conclusion}

Même s'il n'a pas été directement question ni de prototype ni de sens dérivationnel dans les pages qui précèdent, ces notions étaient présentes en filigrane. Nous discuterons brièvement de la pertinence de la notion de «prototype » quant il s'agit de faire une analyse discursive et textuelle. Kleiber (1990: 26] adopte la « règle frégéenne de la détermination de la référence par le sens » qui implique, d'une part, que l'appartenance à une catégorie est conditionnée par le partage de conditions nécessaires et suffisantes (CNS), et, d'autre part, que, pour être en mesure d'employer un mot, il faut en connaître le sens. Les CNS ne sont donc pas des traits «objectifs » mais des traits qui prennent en considération les locuteurs, la culture, etc. Cette approche du sens, autonome et détaché de la référence au réel ${ }^{13}$, érige en principe l'opposition des catégories. La question n'est donc plus « qu'est-ce-qu'un verbe ? », mais « en quoi le verbe diffère-t-il des autres catégories, en particulier de celle du nom ? » On pourrait répondre que le verbe entretient un rapport serré avec un sujet thématique / topic, ce qui revient à centrer la réflexion non plus tant sur l'appartenance catégorielle que sur la prédication. Nous nous garderons de contraster le verbe et le nom en terme de «contenu prédicatif» et aussi étant donné l'importance du «trafic intercatégoriel » verbe - nom. Et, comme Kleiber [1990: 51] le fait dans la version étendue du prototype, plutôt que de considérer que tous les membres d'une catégorie présentent des CNS communes à tous, nous adoptons l'idée que «c'est une ressemblance de famille qui les regroupe ensemble ». Parmi les six critères que développe Kleiber, nous retiendrons ici les deux derniers :

5) L'appartenance à une catégorie s'effectue sur la base du degré de similarité avec le prototype ;

6) Elle ne s'opère pas de façon analytique, mais de façon globale ${ }^{14}$.

Les membres d'une catégorie n'ont pas un degré d'appartenance plein et égal. Un verbe, dans le système langue, est plus ou moins proche du modèle prototypique, plus ou moins verbe. Kleiber [1990: 54] définit la « ressemblance de famille » comme

la structuration qui permet aux membres d'une catégorie d'être reliés les uns aux autres sans qu'ils aient une propriété en commun qui définisse la catégorie.

Il n'y a donc plus de prototype mais des effets prototypiques : la catégorisation est alors justifiée par des "liens d'association» entre les différents membres et non par un rapport entre les différents membres et le protopype. Les rapports entre le centre et la périphérie ont changé. La notion de « ressemblance de famille » s'accompagne d'un glissement de la notion de catégorie à celle du sens d'un terme pour ce qui est des mots polysémiques. Il s'agit de décrire les relations entre les différentes acceptions du mot :

\footnotetext{
${ }^{13}$ La structuration anthropomorphique du sens est commune aux théories culiolienne et guillaumienne.

${ }^{14}$ Les quatre premiers critères que définit Kleiber sont les suivants :

1) La catégorie a une structure interne protopypique ;

2) Le degré de représentativité d'un exemplaire correspond à son degré d'appartenance à la catégorie ;

3) Les frontières des catégories et des concepts sont floues ;

4) Les membres d'une catégorie ne présentent pas des propriétés communes à tous les membres; c'est une ressemblance de famille qui les regroupe ensemble.
} 
Il y a des liens entre les différents sens qui sont tels qu'aucun trait commun n'est exigé. La seule contrainte est que tout sens partage au moins une propriété avec une autre. [Kleiber $1990: 179]$

Cette approche décentrée, non dérivationnelle, de la polysémie lexicale nous paraît probante, cependant, si on s'accorde à penser que le sens est avant tout un objet mental, il est nécessaire d'y ajouter la dimension cognitive et psychologique : connaître le sens d'un mot, c'est aussi être dans un certain état psychologique, c'est le faire sien. On touche là aux limites du système-langue, ou, en tout cas, on ressent le besoin de l'assouplir, de lui conférer une dimension «discursive-variationniste». Avant d'aller plus avant dans ce sens, nous ajouterons que ce qui vient d'être dit d'unités lexicales est transférable aux constructions. Les archétypes conceptuels associés aux constructions comme « la construction attributive permet de conceptualiser l'état ou l'aboutissement du changement d'état», "la construction prépositionnelle et, dans une certaine mesure, la construction avec particule adverbiale permet de conceptualiser le changement d'état en tant que processus ", sont des valeurs ou des effets prototypiques. Énoncer ce que semble être la valeur la plus représentative d'une construction relève de l'idéalisation.

Pourquoi ce long détour par la prototypicité selon Kleiber et son application aux lexèmes polysémiques? Pour dire que «le sens protypique des unités lexicales dans un discours donné » est à " calculer » en fonction du type de discours. Dans BISG, le sens prototypique de feel est celui d'un verbe copule dans la construction attributive. Nous sommes loin de la construction $\mathrm{S} \mathrm{V} \mathrm{O}$ et du toucher tactile. Le sens prototypique de l'unité dans le texte pris comme corpus est en fonction du type de discours ; ce qui revient à admettre la relativité de la notion de prototypicité. Comment pourrait-on garantir la primauté du schéma S V O, le sens de perception tactile de feel, à titre d'exemple, dans ce type de discours ? Nous revenons à notre point de départ: le style grammaticalisé ; le type de discours, la textualité ont une influence sur la "prototypicité relativisée ", la prototypicité des unités en discours. Subjectification et perte de poids lexical sémantique sont systématisés dans l'écriture de l'intime à notre époque. Le genre a-t-il sa propre grammaire verbale ?

\section{Bibliographie}

AdAM Jean-Michel, Linguistique textuelle. Des genres de discours aux textes, Paris, Nathan Université, 1999.

Adam Jean-Michel, Les Textes: Types et Prototypes. Récit, Description, Argumentation, Explication et Dialogue, Paris, Nathan Université, 1992.

KLEIBER Georges, La sémantique du prototype. Catégories et sens lexical, Paris, Presses Universitaires de France, 1990.

LANGACKER Ronald, Concept, Image, and Symbol. The Cognitive Basis of Grammar, Berlin/New York, Mouton de Gruyter, 1990.

LECERCLE Jean-Jacques, La violence $d u$ langage, Paris, Presses Universitaires de France, 1996.

Meschonnic Henri, Critique du rythme : Anthropologie du langage, Lagrasse, Verdier, 1982.

Meschonnic Henri, Ethique et politique du traduire, Lagrasse, Verdier, 2007.

PaUlin Catherine, "Interénonciativité dans "A Lady of Letters" et "Soldiering On" de Alan Bennett, dans Talking Heads », BBC Books, Actes du colloque international "Le destinataire dans les écrits intimes », Editions Universitaires de Dijon, Textes réunis et présentés par CRINQUAND Sylvie, 2001 : 49-57. 
Paulin Catherine, "Lexture linguistique de Faces in the Water de Janet Frame : et si le texte était à la folie ce que l'extra-lucidité est au délire ? », Actes du colloque international «Frontières et syncrétisme », Série Littérature et Histoire des Pays de Langues Européennes, $n^{\circ} 61$, Presses Universitaires de Franche-Comté, Textes réunis et présentés par BEN ABBEs Hédi, 2002 : 23-39.

Paulin Catherine, "L'implicite dans "Swans" de Janet Frame : lecture linguistique », in LEPALUDIER Laurent (éd), L'implicite dans la nouvelle de langue anglaise, Presses Universitaires de Rennes, 2005 : 43-55.

Paulin Catherine, «Le journal intime de Sylvia Plath (Décembre 1985 - Novembre 1959) : un medium malaisé », in DAHAN-GAIDA Laurence (dir.), Actes du colloque international «Le tiers », Série Littérature et Histoire des Pays de Langues Européennes, Presses Universitaires de Franche-Comté, 2007 : 165-180.

PusteJovsky James, The Generative Lexicon, Cambridge Massachusetts, Massachusetts Institute of Technology, 1998.

SEARLE John, Expression and Meaning, Cambridge, Cambridge University Press, 1979.

TESNIERE Lucien, Eléments de syntaxe structurale, Paris, Klincksieck, (1959) 1966.

TourniEr Jean, Précis de lexicologie anglaise, troisième édition, Paris, Nathan Université, 1993. 


\section{Annexe 1 \\ Construction du sens et transfert catégoriel : verbes dénominaux et autres types de conversion}

\section{Verbes dénominaux :}

1. Poison, p. 6 : There's always the chance that I might be poisoning but I couldn't just let the little bastard virus sit and multiply. (Autre type)

2. Arse, p. $23:[\ldots]$ you are probably just busy or can't be arsed to e-mail. (Autre type)

3. Balloon, p. $23:[\ldots]$ I have ballooned to inhuman proportions.

4. Balloon, p. 32 : [...] I have ballooned further. (Relation de comportement: BEHAVE LIKE)

5. Blitz, p. 31 : I could have had it all blitzed in one go. (Les médecins et les traitements) (Relation de comportement : BEHAVE LIKE)

6. Block out, p. 62 : When your loved one goes, either you want to block it out, or you want to hang onto whatever you can... (Relation de mouvement : REMOVE)

7. Bloom, p. 45 : So now I have strict instructions that if anyone asks I am to say you are soon to be blooming with rude health. (Relation de comportement : BEHAVE LIKE)

8. Book, p. 27 : Have booked holiday to Majorca.

9. Book, p. $31:[\ldots]$ have booked a room at a restaurant in St John Street.

10. Book, p. $31:[\ldots]$ am booked in for CT scan of my chest.

11. Book, p. 36 : Shall I book somewhere?

12. Book, p. 80 : So we [...] booked a flotilla holiday in the Sporades... (Autre type)

13. Book up, p. $36:[\ldots]$ it gets all booked up. (Autre type + télicité)

14. Bugger up, p. $50:$ By the time you've buggered up the morning, you might as well finish off your husband's birthday cake for lunch. (Relation de comportement : BEHAVE LIKE)

15. Burden, p. 62 : Anyway, I'm not writing this to burden you - that's the last thing you need. (Relation de mouvement : PUT INTO)

16. Cop, kop, p. 20 : You can wear it after I've kopped it.

17. Cop, kop, p. 20 : I would love to have your underwear after you've copped it. (Relation de comportement : BEHAVE LIKE ?)

18. Clutch, p. 21: So I'm not clutching on to the miracle cure theory. (Relation d'instrument : USE)

19. Crop, p. 39 : All right, the chemotherapy means you have to hoover your hair off the pillow every morning, but it's finally forced you to have your bush cropped, which would look fabulously Jean Seberg if you weighed less than 10 stone. (Relation de mouvement: REMOVE)

20. Dust, p. 85 : However I need only to refer to you to one of the pregnancy manuals dusting up my shelves... (Relation de mouvement : PUT ON)

21. End, p. 29 : Perhaps it's better to end one's life as a vegetable, rather than in pain. (Autre type)

22. End up, p. $78:[\ldots]$ I ended up with a brain tumor, despite confident declarations from all and sundry that I was on the mend. (Autre type)

23. Face, p. $8:$ I couldn't face dealing with sorrow.

24. Face, p. 15 : Cut the crap. Face the music.

25. Face, p. 21 : (let's face it, so much has been wrong there must be a chance of this one). (Il s'agit d'un traitement)

26. Face, p. 31 : But let's face it, the temple has been completely desecrated already.

27. Face, p. $54:[\ldots]$ but can't face any more treatment. (Autre type) 
28. Face up to, p. $21:[\ldots]$ they don't know what you really have to face up to. (Autre type) 29. Favour, p. 21 : I favour more optimistic targets. (Autre type)

30. Fob off, p. 31: They haven't been sitting on any results, it just took two weeks to convince me I needed a CT scan because X-rays aren't sensitive enough. I think from now on they won't fob me off. (Relation de mouvement : REMOVE)

31. Fret, p. 23 : The problem with you being ill is that when I don't hear from you, I fret and worry, almost completley needlessly. (Relation de comportement : BEHAVE LIKE)

32. Garden-ING, p. 63 : Beyond the day mark I think it would have been like a weekend pottering about, gardening, going to our favourite pub. (Autre type)

33. Gun down, p. 29 : Anyway, more fucking treatment looms, though not before I gun down the entire oncology department. (Relation d'instrument : USE)

34. Grieve, p. 61 : Make yourself real in your letters to your children - they will grieve, they must grieve, but by knowing you as a real person, warts and all, it will help their recovery... (Autre type)

35. Harbour, p. 21 : That said, I do harbour a number of fantasies. (Relation de mouvement : PUT IN)

36. Head off, p. 68 : (I think you headed off to the High, while I went to Bishop Kirk and Abingdon). (Relation de mouvement)

37. Hobnob, p. $11:[\ldots]$ you are the only person I know who hobnobs with royalty in quack waiting rooms. (Autre type)

38. Hoover, p. 39 : All right, the chemotherapy means you have to hoover your hair off the pillow every morning... (Relation d'instrument : USE)

39. Kid, p. 32 : I kid you not. (Relation de comportement : BEHAVE LIKE)

40. Pack in, p. 39 : Still, it ain't over till the fat lady's thin. Or until her liver packs in. Or something. (Autre type)

41. Peg, p. 34 : The Movie would be great. Maybe you should make it after I've pegged it. (Autre type)

42. Peter out, p. 32 : So your mad last para remains unread - aren't endings a bugger? I always peter out oddly. (Relation de comportement : BEHAVE LIKE)

43. Pin, p. 66 : I have your articles pinned on my wall in the office as they keep me going. (Relation d'instrument : USE)

44. Pump, p. 34 : Do you really have to take one of your drugs with grapefruit? Sound dangerously complementary to me. Mind you, Guy's are pumping me full of yew tree needles. (Relation d'instrument : USE)

45. Ram, p. 38 : You ram a non-organic carrot up the arse of the next person who advises you to start drinking homoepathic frogs' urine. (Relation d'instrument : USE)

46. Rule out, p. 35 : Sadly, I am a Top London Atheist but death-bed conversion to Judaism not yet ruled out. (Relation de mouvement : REMOVE)

47. Snog, p. 33 : Now I am very worried about who else I may have inadvertently snogged Michael Cook? (Autre type)

48. Spirit off, p. 80 : I was eighteen and in my first year of UCL when Dad was first spirited off to hospital under mysterious circumstances. (Relation de mouvement : PUT INTO)

49. Stomach, p. 77 : I embarked on a weekly Chinese herb and acupuncture regime (£50 a week), which involved wolfing 24 horse tablets a day and drinking as much horrid tea as I could stomach. (Relation de mouvement : PUT INTO)

50. Toy, p. 49 : Instead of toying with a little home-made broth at lunchtime, I have developed an obsession for crisps. (Relation de comportement : BEHAVE TOWARDS)

51. Update onseself, p. 65 : I've tried to get hold of series 4 of E.R., so you could update yourself on the romantic shenanigans of gorgeous George and Nurse Hathaway. (Autre type) 52. Water, p. 59 : [...] Matt never waters the garden... (Relation de mouvement : PUT ON) 
53. Wheel, p. 72 : On really bad days, a listless young man would also be in the basement waiting area, wheeled to and from the ward by orderlies wearing rubber aprons and gloves, which I interpreted as cancer plus some God-awful infectious disease. (Relation d'instrument : USE)

54. Woolf, p. 77 : I embarked on a weekly Chinese herb and acupuncture regime ( $£ 50$ a week), which involved wolfing 24 horse tablets a day and drinking as much horrid tea as I could stomach. (relation de comportement : BEHAVE LIKE)

\section{Autres conversions :}

1. Go, N, p. 18 : But I'd like to straight there from the Departure lounge, and not pass go or waste time with a funeral.

2. Lock down, N, p. 22 : There's been a lock-down here and no mail going in and out for a week, but am hoping this will get out.

3. Odd, N, p. 45: I have seen so many people defy the odds but make no mistake you have a steep hill in front of you but you can reach the summit.

4. Out oneself, V, p. 23 : Have you outed yourself yet?

5. Pay-back, N, p. 49 : I was hoping for a little pay-back...

6. Hoot-ING, N, p. 15 : How is your hooting going? I thought the Kathy Acker piece was a stream of consciousness mess which couldn't win anyone over to her cause - no evidence, just her declaration that 'I'm cured' - nine months after diagnosis, with no objective assessment?

7. 10. Sigh, advice, need, care N, p. 61 : a sigh of relief, my advice, their primary need, loving care

8. Wonder, N, 71 : And my popularity wasn't just a one-month wonder.

\section{Conversions verticales :}

p. 36 : A Fashion Victim Writes retired due to illness, but am coming back as A Cancer Victim Moans. That will be interesting.

p. 52 : I hate you are rendered powerless: that it pulls all the strings and you, Ms-in-controlof-her-life-Picardie, control nothing.

p. 59 : Still, I'm trying to look on the bright side. [...] And it's one way of solving the postfeminist, double-barrelled what-surname-to-give-the-kids dilemma. (His. Who wants to be named after a dead psot-feminist?) 


\section{Annexe 2}

Sens contructionnel des verbes de perception : feel, look, sound et sens constructionnel des verbes de mouvement : go, turn. Expressions périphrastiques vs. verbes simples

Feel :

+ syntagme adjectival :

p. $2:[\ldots]$ the antibiotics started working within 24 hours and I now feel fine.

p. 2: Imagine four days of the worst hangover combined with the worst flu, where you can hardly move, feel poisoned, and half-asleep but not pleasantly out of it all the time. Felt too wretched even to listen to the radio.

p. 2 : I was only sick once (different anti-emetics), had a smaller dose, but it felt worst (cumulative effect).

p. $12:[\ldots]$ she is feeling less weepy today (Sunday).

p. 29 : Please feel free to be rude about him.

p. 35 : I feel pretty well, other than tired and downhearted.

p. 51 : So, it looks like curtains this year. Death seems unreal since I feel relatively well.

p. 51 : Not really anything left in the treatment bag, which means it's probably curtains this year, which still feels unreal since I feel relatively well.

p. 53: I veer between weeping, feeling totally emotionally and physically drained and being unable to believe I am going to die this year...

p. 54 : Doesn't make you feel groggy in the morning... (Temazepam, a drug)

p. 56 : I feel a bit awkward about writing this to you, and it's not my style.

p. 57 : Still, I'm pretty scared. Not that breast cancer has been a picnic so far: all those hats I made people buy when I thought I was going bald and then felt guilty about not wearing. p.60 : I've just read your article 'Before I Say Goodbye' in the Observer today and feel so moved I must write to you.

p. 62 : I felt so glad that you had kids.

p. $65:[\ldots]$ do feel free to contact me.

p. 66 : I became completely certain that they went on in an afterlife - a life I'd never believed in before? Even after nearly 5 years I remember how strongly I felt their spirit with me - it has made me feel far less frightened of death...

p. 72 : Unfortunately, all this suffering didn't make me feel better about my state of health, or fill me with sympathy for others, but made me feel sick, unheroic and afraid.

p. $73:[\ldots]$ but can I be your best friend again so everyone will feel sorry for me at the funeral?

p. 73 : I guess most of my friends simply feel desperately sorry for Matt, me and the children...

+ syntagme nominal :

p. 61 : I still feel loss that I do not have a 'real' mother but also aware that I have unwittingly turned her into a paragon of virtue, a perfect version of motherhood - but forever younger than me.

p. $17:$ ([...] Now I accept the reality of the situation, it's not such a disaster.)

I felt the same about infertility - that hoping and praying for a baby was disempowering and that by saying, 'We can't have kids' I took control and could let go of some of the grief. [...] I know this isn't your approach at all, but I hope you can understand why I feel this way right now. 
+ contenu propositionnel :

p. $18:[\ldots]-$ you wouldn't believe the number of acquaintances who suddenly want to be your best friend and feel they are entitled to regular, blow by blow accounts of your emotional / physiological state.

p. 40 : I grew to love you and feel you were the sister I never had.

p. 45 : I felt I had to write to say how much I admire your courage.

p. 46 : I feel we denied her the chance to get better because we denied her feelings and fears.

p. 54 : Much better than Mogadon, which makes you feel as if you've been hit over the head by a frying pan.

p. 56 : Please forgive me if this letter is crass or a load of presumptuous cheek - it was simply an impulse I felt I should follow.

p. 61 : My advice (I feel that is too demanding but can't think of another word) is to make sure you continue to be allowed to live after your death - however uncomfortable this may be for your partner and family.

p. 64 : Everyone who loves you, will love you still. Everyone who lives you have touched, will feel their lives diminished by your passing.

p. 66 : I became completely certain that they went on in an afterlife - a life I'd never believed in before? Even after nearly 5 years I remember how strongly I felt their spirit with me - it has made me feel far less frightened of death...

p. 80 : I was so moved by your frank and amusing article in Life recently, that I I felt I had to write and impart my experience as one of the 'deserted survivors' that may offer some solace to you and your family.

\section{Look :}

+ Syntagme adjectival :

p. 12 : Shall I make a silly film to show on BBC2 after our deaths? Over-exposed and black and white to make us look georgeous, lots of slo mo running on the beach and Tom Cruise and Nicole Kidman narrating our e-mail correspondence.

p. 25 : But your house always looks fabulously chic and clean.

p. 27 : Looked at my bone scan again which looked 'hot' in the right place.

p. 48 : 'You look so fat.'

p. 48 : 'To make me look less greedy.

p. 48 : 'You look so well.' Actually I don't look particularly well.

+ Particule adverbiale :

p. 59 : And, looking back, I don't have many regrets.

\section{Sound :}

p. 19 : You didn't sound your normal chipper self.

p. 76 : All this (complementary alternative treatment) may sound ridiculous. 
Go, turn :

Go + Adjectif prédicatif :

p. 57 : Still, I'm pretty scared. Not that breast cancer has been a picnic so far: all those hats I made people buy when I thought I was going bald and then felt guilty about not wearing.

p. 58 : Turning into a bruised lemon is, I reckon, better than going mad.

Go + Particule adverbiale :

p. 29 : Well, after weeks of hassling (by me) and faffing (by Guy's) it turns out I do have skull 'involvement'...

Turn + INTO GN :

p. 31 : I was only joking about turning into a vegetable, but I am starting to want to know exactly how one dies of breast cancer.

p. 38 : As you'd expect, the diagnosis turns you into a grumpy, bitter, envious old cow.

p. 39: Abruptly, you enter the bleaky euphemistic world of palliative care. Pollyanna commits suicide. Your chic crop turns into a toilet brush.

p. 58 : Turning into a bruised lemon is, I reckon, better than going mad.

p. 77 : And even though I turned down his offer of low-level oxygen administration..., the treatment turned into a huge pressure.

p. 38 : As you'd expect, the diagnosis turns you into a grumpy, bitter, envious old cow.

+ Particule Adverbiale :

p. 27 : Interviewed Robbie (to his friends) for Style; became slightly in love. He turned out to have shrunk.

p. 29 : Well, after weeks of hassling (by me) and faffing (by Guy's) it turns out I do have skull 'involvement'.

p. 72 : I can't believe my popularity is simply due to the fact that I'm not yet looking as scary as my fellow patients... Nor can it be my former niceness, which never used to cut it when turning up for birthday dinners.

p. 77 : And even though I turned down his offer of low-level oxygen administration..., the treatment turned into a huge pressure.

p. 82 : [...] time having run out.

\section{Feel/look/sound like :}

p. 12 : Sometimes I feel like putting a message on the answerphone.

p. 31 : I feel like I'm fighting the NHS the whole time.

p. 2 : [...] since he is not at all attractive though very funny and looks like Dr. Green in ER.

p. 14 : Come and stand next to me and you'll look like you're in profile.

p. 20 :But I look like such a slob most of the time.

p. 34 : [...] unexpected questions... (such as do I look like my mum?)

p. 34 : We've borrowed a HI-8 Sony video camera from friends who live in Vietnam and buy state of the art electronic goods in Singapore, so the kids will know what I looked like (tired and self-conscious).

p. 79 : For just $£ 250$, I started looking like a film star. 
p. 87-88 : I'm so fat at the moment - the steroids make me look like I have a goitre...

p. 94 : (lettre manuscrite à son fils) You are nearly two and the sweetest boy in the whole world. You look like an angel.

p. 95 : (lettre manuscrite à sa fille) You look just like me. Hooray!

p. 20 : (Yuck, I sound like a perv)

Autres périphrases verbales :

p. 7 : I think I'd rather go nuts than die in agony.

p. $17:([\ldots]$ Now I accept the reality of the situation, it's not such a disaster.)

I felt the same about infertility - that hoping and praying for a baby was disempowering and that by saying, 'We can't have kids' I took control and could let go of some of the grief.

p. 94 : (lettre manuscrite à son fils) You are the best thing that ever happened to me and Daddy and the hardest thing to let go.

p. 58 : Great. I'm going to die, but I'm going to go bonkers first.

p. 87 : The Gower was fab, though started going a bit bonkers and having Prozac-induced panic attacks in the supermarket.

p. 63 : The thought that you want them to be happy - as I know my David wanted me to - will be the real challenge for your family early on. But reading your article and trying to imagine your family, they will be up to it, even if it takes time. And time is what they have.

p. 64 : Gary said 'you know me mum, I'm a bit dotty.'

p. 65 : If there are any other progs from $\mathrm{C} 4$ that you'd like me to lay my hands on, do feel free to contact me.

p. 66 : I think that she felt the Observer had somehow become a medical journal and was to be hidden at all costs from anyone who might throw a wobbler if they caught sight of the article. p. 66 : I have your articles pinned on my wall in the office as they keep me going.

p. 67 : I am hoping to do my bit in good old East Yorkshire.

p. $68:[\ldots]$ but I hope that the knowledge of the impression you made today might slighly lift your spirits.

p. 71 : People I haven't seen for years for years want to take me out for lunch.

p. $78:[\ldots]$ I ended up with a brain tumor, despite confident declarations from all and sundry that I was on the mend.

p. 82 : It's very expensive this stuff (aftershave) you know. I'm hoping to finish it before I kick the bucket. 